\title{
Multiple Cysten an der Irishinterfläche und am Corpus ciliare.
}

\author{
(Pseudomelanosarkom des Corpus ciliare.) \\ Von \\ Dr. Adolf H. Pagenstecher, \\ Augenarzt in Wiesbaden.
}

Im Jahre 1906 demonstrierte Wintersteiner auf der Versammlung der ophth. Gesellschaft in Heidelberg Präparate von primärer Cyste der Irishinterfläche. Der Fall war der erste seiner Art und ist sowohl klinisch als auch pathologisch-anatomisch sehr interressant. Seit längerer Zeit bin ich im Besitz eines Bulbus mit der gleichen Erkrankung und bei der grossen Seltenheit der Affektion dürfte eine kurze Beschreibung wohl angezeigt sein.

Herr H., etwa 60 Jahre alt, stellte sich am 13. IX. 06 zum ersten Male in unserer Privatklinik zur Untersuchung vor mit der Angabe, dass er mit dem rechten Auge sehlechter sehe. R. $S=\left.{ }^{6}\right|_{36}-\left.{ }^{6}\right|_{24}$ mit $-3,0 D$. Jg. 7 mit $+2,0 D$. L. $S={ }_{6}^{6}$. Jg. 1 mit $+2,0 D$.

Diagnose: Opacitates lentis o. $d$.

Die Untersuchung bei erweiterter Pupille ergab, dass die Trübungen unten aussen am stärksten waren.

Fundus normal.

Am 14. III. 1907 stellte sich der Patient wieder vor mit der Angabe, dass beim Lesen sehr häufig starke Beschwerden im rechten Auge auftreten; er habe im Ausland einen Augenarzt konsultiert, der ihm dringend die Entfernung des Auges empfohlen habe.

$\mathrm{Zn}$ gleicher Zeit kam ein Brief des konsultierten Ophthalmologen (Professor van Duyse in Gent), in dem die Ansicht ausgesprochen war, dass es sich um einen von der Irishinterfläche ausgehenden malignen Tumor handele, und dass das Ange sofort enucleiert werden müsse.

Status: Rechtes Auge. Bulbus ganz reizfrei. Unten aussen ist in einer Ausdehnung von $3 \mathrm{~mm}$ die vordere Kammer sehr seicht, die Iris ist stark nach vorne gedrängt. Sonst ist sie normal. Keinerlei Zeichen von Entzündung. Die Linse ist unten aussen getrübt; zwischen ihr und der nach vorne gedrängten Iris sieht man aus der hintern Kammer eine schwarzbraune Geschwulst hervorragen. Dieselbe ist am Pupillarrand (bei etwa mittelweiter Pupille) $2-3 \mathrm{~mm}$ breit und überragt denselben um $1 \mathrm{~mm}$. Die Lage dieser Geschwulst entspricht der Linsentrübung. Glaskörper und Fundus normal. R. $S={ }^{6 / 36}-6 / 24$ mit $-3,0 D$. Jg. 7 mit $+2,0 D$. Spannung normal. Urin frei von Alb. und Saceh. 
Linkes Auge normal.

Diagnose: Sarkom des Corpus ciliare oder Tumor von der Irishinterfäche ausgehend.

Es wurde dem Patienten die Enucleation proponiert und dieselbe am nächsten Tage in Lokalanästhesie ausgeführt. Glatte Heilung. Der Bulbus war in Formol fixiert worden; er wurde durch einen von oben innen nach unten aussen gehenden Schnit,, der die prominente Stelle der Iris traf, in 2 Hälften geteilt.

Makroskopisch war kein Tumor zu sehen, die Iris war an der Stelle, wo er vermutet war, etwas nach vorne gedrängt. Einbettung in Celloidin.

Mikroskopischer Befund: Cornea normal.

Die Iris ist in ihren vordern Schichten normal; das Gewebe ist klar; nirgends Zeichen von Entzündung. An der Stelle, wo intra vitam die Vorbucklung war, ist der Kammerwinkel aufgehoben.

Die Iris liegt hier stellenweise eine kurze Strecke weit der Cornea an. Ihre Vorderfläche lässt auch $\mathrm{da}$, wo sie die Descemetsche Membran nicht berührt, die normalen Krypten vermissen. Die Pigmentsehieht der Iris ist in ihre 2 Blätter gespalten, die Spaltung beginnt an der Iriswurzel, reicht aber nieht ganz bis zum Pupillarrand, sondern endigt in einiger Entfernung von ihm; hier hat die abgelöste hintere Pigmentschicht sich um $180^{\circ}$ gedreht und überragt den Pupillarrand. Sie endigt dann frei in der Kammer zwischen der hier von der Linse abgedrängten Iris und linse.

Der andere Teil des hintern Pigmentblattes liegt auf der vordern Linsenfläche auf. Er beginnt nahe dem oben erwähnten freien Ende und bedeckt etwa ${ }^{1 / 4}$ der vordern Linsenkapsel, der er aufliegt, geht dann an den Zonulafasern entlang zum vordern Teile des Corpus ciliare, um an der Iriswurzel zu endigen. Trotzdem die Kontinuität des hintern Pigmentblattes nicht erhalten ist, besteht nach der ganzen Sachlage kein Zweifel, dass sie intra vitam vorhanden war und die so zwisehen beiden Pigmentblättern gebildete Cyste beim Durchschneiden des Bulbus geplatzt ist.

Nicht in allen Schnitten liegt die hintere Wand dieser Cyste der Linse fest auf, sondern ist öfter aufgerollt in der vergrösserten hintern Kammer. Das Pigmentblatt, das die hintere Blasenwand bildet, hat seine Konturen etwas eingebüsst, ist abgeplattet und scheint etwa so dick wie die vordere linsenkapsel. Ein Pigmentverlust hat nicht stattgefunden.

In andern Schnitten, wo die Cyste peripher getroffen wurde, ist das Pigmentblatt nur an der Iriswurzel gespalten. Es reicht dann die Wand nicht bis zur Linse, die Iriskrypten sind deutlich und der Kammerwinkel ist fast normal konfiguriert. Innerhalb der Cyste liegen stark pigmentierte Kugeln, die sich aus abgestossenen Pigmentzellen gebildet haben.

Ausser dieser grossen Cyste findet man auch an andern Stellen die Pigmentschicht gespalten, so z. B. an der der grossen Iriscyste gegenüberliegenden Partie. Hier handelt es sich offenbar um beginnende kleine Cysten; sie liegen entweder hart an der Iriswurzel oder in der Mitte der Irishinterfläche an, nie in der Nähe des Pupillarrandes.

Das abgehobene Pigment ist bei diesen kleinen Cysten in seiner Form gut erhalten.

Der Ciliarkörper lässt nirgends Zeichen von Entzündung erkennen; 
an einzelnen Stellen weisen die Blutgefässe eine leichte beginnende hyaline Degeneration auf.

Mannigfaltig sind die Veränderungen an seinem Epithel. An ihm finden sich grössere und kleinere Cysten, einzeln oder in Gruppen zusammenliegend; die kleinsten werden dadurch gebildet, dass eine Membran, die aus gut konfigurierten Epithelzellen besteht, von einem Ciliarfortsatz zum andern zieht. Wird die Blase dann grösser, so dehnt sich die nach dem Bulbusinnern liegende Wand, die Zellen werden flacher und man kann so alle Übergangsformen bis zu lang ausgezogenen oder endothelartigen Zellen mit länglichem Kern erkennen. Diese oft sehr dünne Wand geht dann in das Epithel des Ciliarkörpers über, bei den grösseren Cysten ist die Höhe eines Ciliarfortsatzes oft zu einer Spitze ausgezogen.

Während einzelne Cysten durch eine einfache Spaltung der Pars cil. ret. in ihre 2 Blätter entstanden sind, begrenzt bei andern allseits das unpigmentierte den Hohlraum. Es hat an vielen Stellen eine starke Proliferation des Ciliarepithels stattgefunden und man muss wohl annehmen, dass nach Ablösung des innern Blattes das Pigmentepithel von einer neugebildeten Sohicht unpigmentierten Ciliarepithels überzogen wurde. Oft bilden sich die Hohlräume auch in dem gewucherten Epithel und man sieht zuweilen 5-6 verschiedene grosse Cysten, die von recht verschieden gestalteten Zellen, deren direkter $\mathrm{Zu}$ sammenhang mit dem Ciliarepithel deutlich nachzuweisen ist, begrenzt werden. Wo die Cystenwand schief getroffen ist, kann man zuweilen reichlich Zellen mit rundlichem oder ovalem Kern, ohne deutliche Zellgrenzen erkennen. Auch die nach dem Bulbusinnern zugelegene Cystenwand zeigt an einzelnen Stellen Pigment, ohne dass ein direkter Ubergang ins Pigmentepithel besteht. Die Cystenbildungen sind nicht nur in den der grossen Iriscyste anliegenden Partien, sondern finden sich fast über das ganze Corpus ciliare verteilt.

An der Linse, die nicht disloziert ist, sind am Äquator die Fasern gequollen und zerfallen, besonders in der unten aussen gelegenen Partie.

Die Retina zeigt die Veränderungen, die man als Iwanoffsches Ödem bezeichnet, in hohem Grade ausgeprägt. Sonst ist sie normal und liegt iberall gut an.

Die übrigen Teile des Auges lassen nichts Krankhaftes erkennen. Zum klinischem Verlauf wäre noch nachzutragen, dass der Patient sich 1 Jahr nach der Enucleation nochmals zur Untersuchung vorstellte mit der Angabe, er fühle sich seit der Operation viel wohler; früher habe er oft Kopfschmerzen bei der Arbeit auf der rechten Seite gehabt; er habe diese Erscheinung unerwähnt gelassen, da er sie nicht auf das Auge bezogen habe.

Fassen wir den pathologisch-anatomischen Befund zusammen, so handelt es sich um eine multiple Cystenbildung an der Irishinterfläche und auf dem Corpus ciliare. Die Cysten der Tris kommen lediglich durch Trenunng der beiden Pigmentblätter zustande; am Ciliarkörper entstehen sie entweder durch Loslösung des unpigmentierten Epithels oder sie bilden sich wohl auch in dem proliferierenden Epithel selbst; ibre Wand ist meist nicht pigmentiert. 
Während in den Fällen von Wintersteiner deutlich Symptome, die auf Glaukom schliessen liessen, den Kranken zum Arzt trieben, und auch klinisch Drucksteigerung festgestellt wurde, hatte unser Patient nur allgemeine Klagen, die wohl auf periodische Drucksteigerung zurïckzuführen sind, trotzdem dass das Auge ruhig und die Spannung bei der Untersuchung normal war. Demnach wird man nicht fehlgehen, wenn man annimmt, dass doch zuweilen leichte Erscheinungen von Glaukom vorhanden waren, da die Beschwerden mit der Enucleation ganz schwanden. Ein Diabetes, der eine Tendenz zur leichten Ablösung des Pigmentepithels verständlich gemacht hätte, lag nicht ror.

Die längere Zeit der Beobachtung erlaubt uns einen Rückschluss anf die Schnelligkeit des Wachstums; während bei der ersten Untersuchung bei Mydriasis noch nichts von der Neubildung gesehen wurde, trotzdem die hier gelegenen Linsentrübungen besonders vermerkt sind, überragte die Cyste nach 6 Monaten die mittelweite Pupille um etwa $1 \mathrm{~mm}$; die Linse wurde dabei nicht verdrängt, auch die Trübung kann nicht wesentlich zugenommen haben.

Es erhebt sich die Frage, ob die Enucleation bei richtiger Diagnosenstellung nicht hätte umgangen werden können. (Das klinische Bild war für die Diagnose eines Melanosarkoms ein so charakteristisches, wie man es sich nur denken kann, so dass eine Durchleuchtung mit der Sachschen Lampe nicht vorgenommen wurde.) Wenn man auch die Operation in solchem Falle noch hinausgeschoben hätte, so wäre man doch wahrscheinlich bald zu diesem Eingriff durch die starken Beschwerden des Kranken gezwungen worden, da eine Heilung dieser Affektion, sei es auf operativem oder sonstigem Wege, unmöglich ist.

Bei dem Alter des Patienten ist die Proliferation des Ciliarkörperepithels nichts Besonderes; sie kommt oft vor und kann sogar auf dem Schnitt leicht cystoide Bildungen vortäuschen. Kuhnt und Kerschbaumer haben darauf schon vor längerer Zeit bingewiesen.

Was die histologische Stellung dieser Cystenbildung anlangt, so wird es nicht leicht, sie einzureihen. Um eine maligne Neubildung handelt es sich offenbar nicht und wenn wir das Schema von Fuchs in seiner ausführlichen Arbeit (v. Graefe's Arch. 68, 3) berücksichtigen, lässt es sich dort nicht unterordnen. Bei Berïicksichtigung der klinischen Erscheinungen - schwarzbrauner Tumor aus der hintern Kammer hervorragend, die Tris nach vorn drängend - kann man sie wohl als Pseudomelanosarkom des Corpus ciliare bezeichnen. 